\title{
Priorités, obstacles et facilitateurs concernant le traitement à distance des symptômes du cancer: enquête auprès des infirmières en oncologie du Canada
}

par Gail Macartney, Dawn Stacey,

Meg Carley et Margaret B. Harrison

\begin{abstract}
Abrégé
Une enquête réalisée auprès de 368 infirmières en oncologie du Canada a révélé que $54 \%$ d'entre elles fournissaient un soutien à distance aux patients en oncologie. Les symptômes les plus fréquemment signalés étaient la fatigue, la douleur, la nausée, la constipation et l'anxiété. Les symptômes courants que les infirmières s'estimaient les moins capables de gérer étaient l'anxiété, la neuropathie, la dépression, l'anorexie et les altérations cutanées. La plupart des infirmières convenaient que des lignes directrices étaient nécessaires, que celles- ci devaient être accessibles et améliorer la continuité des soins. Quarantetrois pour cent des répondantes jugeaient que les lignes directrices existantes étaient inadéquates pour les symptômes complexes. Seulement $54 \%$ des répondantes étaient d'accord pour dire qu'elles avaient reçu suffisamment de formation en gestion des symptômes. Les obstacles courants comprenaient les contraintes temporelles, l'obtention au téléphone de renseignements exacts sur les patients, les volumes de travail élevés et des lacunes en matière de connaissances ou de formation. Les facteurs facilitant la fourniture d'un soutien à distance incluaient l'accès aux dossiers de santé électroniques, l'accessibilité des membres de l'équipe, la technologie disponible et la facilité de documentation.
\end{abstract}

\section{Au sujet des auteures}

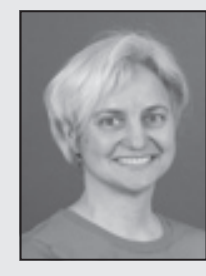

Gail Macartney, IA (cat. spéc.), B.Sc. (sp.), M.Sc.(A), Infirmière praticienne, Division de la Neurochirurgie, Hôpital pour enfants de l'Est de l'Ontario, 401 chemin Smyth, Ottawa, ON K1H $8 L 1$

Doctorante, École des sciences infirmières de l'Université Queen's, 78 Barrie Street, Kingston, ON K $7 L$ SN6

Courriel: gail.macartney@sympatico.ca; Travail: 613 737-7600 poste 2640; Téléc.: 613 738-4228

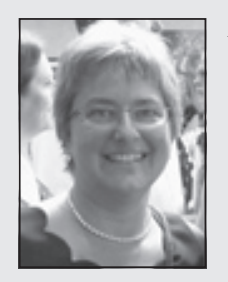

Dawn Stacey, inf., Ph.D., Directrice, Groupe de recherche sur les outils d'aide à la décision pour les patients, Institut de recherche de l'Hôpital d'Ottawa, Professeure agrégée, École des sciences infirmières de l'Université d'Ottawa

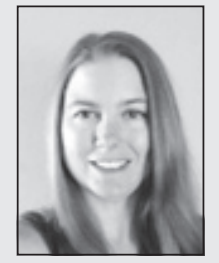

Meg Carley, B.Sc., Gestionnaire de données, École des sciences infirmières de l'Université Queen's, 78 Barrie Street, Kingston, ON K7L 3N6. Gestionnaire de données, Partenariat canadien contre le cancer

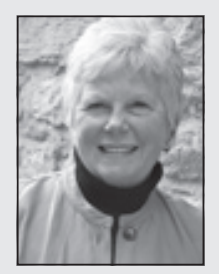

Margaret B. Harrison, inf., Ph.D., Professeure, École des sciences infirmières, Santé communautaire et épidémiologie, Directrice, Queen's Joanna Briggs Collaboration, Scientifique principale, Practice and Research in Nursing (PRN) Group, Université Queen's, 78 Barrie Street, Kingston, ON K7L 3N6

\section{Contexte}

Les services de conseil infirmier par téléphone sont en essor à l'échelle nationale et internationale afin d'améliorer l'accès aux soins de santé (Goodwin, 2007; Holmstrom \& Hoglund, 2007) et de minimiser les coûts (Wilson \& Hubert, 2002). Du fait de l'augmentation du nombre de patients atteints de cancer et du stress placé sur les ressources de cancérologie, les infirmières en oncologie font face à des exigences croissantes concernant la fourniture d'un soutien à distance aux patients et à leurs proches concernant la gestion des symptômes à domicile. Une récente enquête sur la satisfaction menée auprès de 276 patients cancéreux traités en ambulatoire dans un hôpital d'enseignement universitaire en milieu urbain situé à Montréal, Québec, a permis de découvrir que la communication téléphonique avec les professionnels de la santé obtenait les plus faibles appréciations des patients en matière de satisfaction (Richard et al., 2010). Pourtant, la possibilité pour les patients de parler au téléphone aux professionnels de la santé de leurs préoccupations sanitaires a été dégagée comme étant une priorité. Il est important, manifestement, de mieux comprendre ces préoccupations en matière de santé et de déterminer dans quelle mesure les infirmières se sentent compétentes de les aborder à distance.

C'est en général au téléphone que les infirmières en oncologie fournissent un soutien à distance. Divers termes sont utilisés pour décrire la fourniture d'une consultation téléphonique, par un professionnel de la santé, notamment télésanté, services de conseil téléphonique, télépratique infirmière, consultation téléphonique, télétriage et prestation de soins par téléphone. Selon sa définition, la prestation de soins par téléphone englobe la fourniture d'information, de conseils et/ou de soutien (Wilson \& Hubert, 2002); le télétriage, quant à lui, est la démarche qui consiste à faire un dépistage et à recueillir, au téléphone, les symptômes d'une personne ayant appelé afin d'évaluer l'urgence d'un problème de santé (Black, 2007; Stacey, 2003).

L'évaluation et la gestion des symptômes constituent des tâches particulièrement difficiles lorsque les interactions infirmière-patient surviennent au téléphone puisque c'est tout un défi pour les soignants d'évaluer une situation avec si peu d'information sensorielle (Black, 2007). Les infirmières en oncologie doivent dégager une quantité suffisante de renseignements exacts sur des symptômes particuliers, déterminer si des stratégies de gestion des soins autoadministrés suffiront ou si le patient exige une évaluation plus poussée en personne. La dispensation de soutien à distance peut favoriser une utilisation plus appropriée des soins de santé (Mayo, Chang \& Omery, 2002). Quoique les symptômes les plus courants éprouvés par les patients en oncologie traités en ambulatoire aient été signalés dans d'autres études (Barbera et al., 2010), on en sait très peu sur les types de symptômes qui sont mentionnés par les patients dans le cadre des services de santé dispensés au moyen du téléphone. La présente étude avait pour but de cerner les enjeux prédominants et les plus difficiles en matière de gestion des symptômes pour les infirmières en oncologie fournissant du soutien à distance relativement aux symptômes. Elle explorait également les facteurs facilitants et les obstacles éventuels à la fourniture d'un soutien à distance lors de l'évaluation des symptômes, du triage et de la gestion en la matière.

\section{Méthodes}

Devis. Une enquête en ligne a été réalisée entre le 3 septembre et le 27 octobre 2009 auprès d'infirmières en oncologie de l'ensemble du Canada. L'approbation déontologique a été obtenu du Comité d'éthique de la recherche de l'Université Queen's. 
Instrument. L'outil d'enquête a été élaboré en s'appuyant sur une enquête précédente sur les services infirmiers téléphoniques offerts dans le cadre des programmes d'oncologie ambulatoire ontariens (Stacey et al., 2007), sur des enquêtes précédentes sur l'utilisation par les infirmières d'outils fondés sur des données probantes dans la pratique clinique (p. ex. lignes directrices sur les ulcères aux jambes, outils d'aide à la décision pour les patients) (Graham et al., 2001; Stacey et al., 2005), et sur le Modèle d'application de la recherche d'Ottawa (OMRU) (Graham \& Logan, 2004). Les articles de l'enquête ont été modifiés afin de refléter les soins infirmiers en oncologie et le milieu de soins ambulatoires. Les infirmières devaient évaluer 16 symptômes courants liés au traitement du cancer à l'aide d'une échelle Likert en 10 points allant d'extrêmement rare à extrêmement courant pour ce qui est de la fréquence des symptômes. La confiance du personnel infirmier a été évaluée pour ces mêmes symptômes une fois de plus au moyen d'une échelle à 10 points allant de Pas confiante à extrêmement confiante. Une question ouverte a permis de recueillir des ajouts aux 16 symptômes originaux. L'enquête comprenait 19 énoncés qui mesuraient les attitudes des infirmières ainsi que leurs comportements, connaissances, degré de confiance et perceptions sur les obstacles environnementaux liés à l'utilisation des lignes directrices. Les répondantes évaluaient chaque énoncé à l'aide d'une échelle Likert à 5 points s'étendant de tout à fait d'accord à tout à fait en désaccord. Des questions ouvertes demandaient aux participantes de dégager les obstacles à la fourniture d'une gestion des symptômes à distance. L'enquête a également été conçue en vue de recueillir des renseignements sur le comment et sur le quand de la fourniture du soutien à distance y compris les caractéristiques d'infrastructure dans le milieu clinique comme l'accès à des lignes directrices et à une documentation électronique. La validité apparente du questionnaire de l'enquête a été établie par un groupe de 11 chercheurs et praticiens ayant une expertise des soins infirmiers en oncologie et des méthodes de recherche. L'enquête en ligne a fait l'objet d'un test préalable auprès de trois infirmières en oncologie.

Participants. Les personnes suivantes ont été invitées à participer : les infirmières qui savaient lire l'anglais et qui étaient membres de l'Association canadienne des infirmières en oncologie (ACIO/CANO), de la communauté de pratique des infirmières en pratique avancée en oncologie d'Action Cancer Ontario et/ou d'un serveur de liste destiné aux infirmières enseignantes en oncologie du Canada.

Procédures. L'enquête a été administrée en anglais sous la forme d'un questionnaire en ligne au moyen du logiciel d'enquête SurveyMonkey ${ }^{\circledR}$. Les procédures d'administration de l'enquête en ligne étaient basées sur la Tailored Design Method for Surveys (Dillman, 2000). Les infirmières admissibles à la participation ont reçu l'invitation initiale sous forme de courriel qui expliquait le but de l'enquête et fournissait des

instructions, les dates limites et le temps prévu pour le remplissage. On fournissait un lien vers un feuillet d'information donnant davantage de détails et l'accès au questionnaire de l'enquête. L'enquête prenait approximativement 10 minutes à remplir. Selon la démarche de Dillman (2000), des rappels ont été envoyés deux, quatre et cinq semaines plus tard, pour un total de quatre contacts. Les rappels électroniques étaient produits automatiquement pour les personnes qui n'avaient pas encore soumis leur questionnaire. Les données recueillies au moyen de SurveyMonkey ${ }^{\circledR}$ ont été téléchargées dans SPSS $\subset$ d'IBM $\subset$ (version 19 pour Windows).

Analyse. Les résultats démographiques ont été totalisés et décrits au moyen de statistiques unidimensionnelles. Les données ont été décrites relativement à la fréquence (en pourcentage) pour les variables nominales, et à la moyenne, à l'écart-type, à l'étendue et à la médiane pour les variables continues. Pour dégager les priorités sur le plan des symptômes, ces derniers ont été ordonnés de la fréquence la plus haute à la plus basse (1-16) et de la confiance

Tableau 1 : Caractéristiques des répondantes sur le soutien à distance

\begin{tabular}{|c|c|}
\hline Caractéristiques des répondantes & Fréquence (\%) n=197 \\
\hline $\begin{array}{l}\text { Milieu de soins oncologiques pour le soutien à distance } \\
\text { Programme de cancérologie ambulatoire } \\
\text { Soins aux adultes hospitalisés } \\
\text { Soins à domicile/soins communautaires } \\
\text { Soins aux enfants hospitalisés } \\
\text { Autres milieux (p. ex. service des admissions et congés) }\end{array}$ & $\begin{array}{c}121(61,4) \\
23(11,7) \\
7(3,6) \\
1(0,5) \\
15(7,6)\end{array}$ \\
\hline $\begin{array}{l}\text { Affiliations professionnelles* } \\
\text { Membre de l'ACIO/CANO } \\
\text { Membre de la CdP d'IPA } \\
\text { Certification canadienne (CSIO(C)) } \\
\text { Certification américaine (OCN) }\end{array}$ & $\begin{array}{c}140(71,1) \\
21(10,7) \\
110(55,8) \\
8(4,1)\end{array}$ \\
\hline $\begin{array}{l}\text { Études postsecondaires } \\
\text { Diplôme collégial en soins infirmiers } \\
\text { Baccalauréat en soins infirmiers } \\
\text { Autre baccalauréat } \\
\text { Maîtrise en soins infirmiers } \\
\text { Autre maîtrise }\end{array}$ & $\begin{array}{c}104(52,8) \\
107(54,3) \\
13(6,6) \\
50(25,4) \\
11(5,6)\end{array}$ \\
\hline $\begin{array}{l}\text { Années d'expérience en soins infirmiers } \\
\text { > 2-5 années } \\
\text { > 5-10 années } \\
>10-15 \text { années } \\
\text { > } 15 \text { années }\end{array}$ & $\begin{array}{c}4(2,0) \\
13(6,6) \\
14(7,1) \\
135(68,5)\end{array}$ \\
\hline $\begin{array}{l}\text { Années d'expérience en soins infirmiers en oncologie } \\
\quad<1-2 \text { années } \\
\quad>2-5 \text { années } \\
>5-10 \text { années } \\
>10-15 \text { années } \\
>15 \text { années }\end{array}$ & $\begin{array}{c}3(1,5) \\
18(9,1) \\
27(13,7) \\
37(18,8) \\
81(41,1)\end{array}$ \\
\hline $\begin{array}{l}\text { Pratique clinique actuelle en soins infirmiers en oncologie* } \\
\text { Chimiothérapie } \\
\text { Soins de soutien } \\
\text { Soins palliatifs } \\
\text { Radiothérapie } \\
\text { Survivance } \\
\text { Chirurgie } \\
\text { Prévention } \\
\text { Dépistage } \\
\text { Réadaptation }\end{array}$ & $\begin{array}{c}108(54,8) \\
72(36,5) \\
64(32,5) \\
47(23,9) \\
26(13,2) \\
26(13,2) \\
15(7,6) \\
9(4,6) \\
4(2,0)\end{array}$ \\
\hline
\end{tabular}


du personnel infirmier la plus basse à la plus haute (1-16). Cette confiance du personnel infirmier a été représentée par rapport à la fréquence des symptômes ordonnée du bas vers le haut pour chaque symptôme. Dans l'échelle Likert, les réponses aux questions concernant les attitudes, les comportements et les connaissances ont été reclassées selon les catégories d'accord (tout à fait d'accord ou d'accord), en désaccord (tout à fait en désaccord ou en désaccord), et neutre. L'analyse de contenu thématique qualitative a été retenue pour les questions ouvertes à propos des autres symptômes et des obstacles à la fourniture d'un soutien à distance. Les symptômes additionnels ont été classés dans les catégories suivantes : physique, émotionnel/coping, cognitif, personnel, logistique et, autres enjeux généraux.

\section{Résultats}

Sur les 653 répondantes possibles, 56\% $(\mathrm{n}=368)$ ont rempli le questionnaire de l'enquête et parmi elles, 54\% (197/368) fournissaient du soutien à distance à des patients en oncologie [98\% (193/197) au moyen du téléphone; 30\% (60/197) au moyen du courriel]. La répondante type œuvrait au sein d'un programme d'oncologie ambulatoire, était membre de l'ACIO/CANO, détenait la certification en oncologie, avait obtenu un baccalauréat et travaillait

Tableau 2: Caractéristiques de la fourniture du soutien à distance

\begin{tabular}{|c|c|}
\hline & $\begin{array}{c}\text { Fréquence (\%) } \\
n=197\end{array}$ \\
\hline $\begin{array}{l}\text { Heures durant lesquelles les patients peuvent appeler } \\
\text { Heures d'ouverture régulières (dans la journée du lundi au vendredi) } \\
24 \text { h/jour, } 7 \text { jours sur } 7 \\
\text { Fins de semaine et jours fériés } \\
\text { Soirs }\end{array}$ & $\begin{aligned} 175 & (88,8) \\
11 & (5,6) \\
6 & (3,0) \\
2 & (1,0)\end{aligned}$ \\
\hline $\begin{array}{l}\text { Modèle de soins régissant les services de soutien à distance } \\
\text { Soins infirmiers intégraux } \\
\text { Une infirmière est assignée à la gestion de tous } \\
\text { les appels de la journée/du quart } \\
\text { Autre (p. ex. soins infirmiers en équipe) }\end{array}$ & $\begin{array}{l}92(46,7) \\
41(20,8) \\
55(27,9)\end{array}$ \\
\hline $\begin{array}{l}\text { Documentation dans le dossier de santé } \\
\text { des appels y compris les courriels } \\
\text { Oui, systématiquement } \\
\text { Oui, au besoin } \\
\text { Non }\end{array}$ & $\begin{array}{c}124(62,9) \\
49(24,9) \\
15(7,6)\end{array}$ \\
\hline $\begin{array}{l}\text { Format de la documentation } \\
\text { Formulaires papier } \\
\text { Documentation informatisée } \\
\text { Autre }\end{array}$ & $\begin{array}{c}107(54,3) \\
51(25,9) \\
16(8,1)\end{array}$ \\
\hline $\begin{array}{l}\text { Utilisation de protocoles/lignes directrices cliniques pour le triage } \\
\text { ou la gestion des symptômes } \\
\text { Oui } \\
\text { Non }\end{array}$ & $\begin{array}{c}134(68,0) \\
36(18,3)\end{array}$ \\
\hline $\begin{array}{l}\text { Utilisation des protocoles/lignes directrices cliniques } \\
\text { Comme ouvrages de référence } \\
\text { Surtout pour l'orientation des infirmières } \\
\text { Système informatisé intégré } \\
\text { Aucune utilisation } \\
\text { Autre }\end{array}$ & $\begin{array}{l}132(67,0) \\
51(25,9) \\
41(20,8) \\
15(7,6) \\
16(8,1)\end{array}$ \\
\hline $\begin{array}{l}\text { Source des protocoles/lignes directrices } \\
\text { Lignes directrices d'Action Cancer Ontario } \\
\text { L'organisme a élaboré les siens/siennes } \\
\text { Lignes directrices de la B.C. Cancer Agency } \\
\text { Autre }\end{array}$ & $\begin{array}{l}96(48,7) \\
47(23,9) \\
42(21,3) \\
35(17,8)\end{array}$ \\
\hline
\end{tabular}

en soins infirmiers depuis plus de 15 ans (tableau 1). La pratique clinique des infirmières était axée sur l'administration de la chimiothérapie (55\%), les soins de soutien (37\%), les soins palliatifs (32\%) et la radiothérapie (24\%). Sur les 197 répondantes qui fournissaient du soutien à distance, $89 \%$ indiquaient que le soutien était prodigué durant les heures d'ouverture régulières (du lundi au vendredi) et $10 \%$ dispensait un soutien à distance en dehors des heures d'ouverture régulières (p. ex. les fins de semaine, 24 heures sur 24, sept jours par semaine) (voir le tableau 2). La majorité des répondantes documentaient systématiquement les appels, 54\% d'elles au moyen de formulaires papier et $8 \%$ rapportaient qu'elles ne documentaient aucunement les interactions à distance dans le dossier de santé. Deux tiers des infirmières utilisaient des protocoles ou des lignes directrices mais principalement comme ouvrage de référence (67\%). Les lignes directrices utilisées provenaient d'Action Cancer Ontario (ACO) (49\%), de la Colombie-Britannique (21\%) ou avaient été élaborées par leur propre établissement (24\%).

Priorités pour la gestion à distance des symptômes. Les cinq symptômes les plus souvent mentionnés étaient: la fatigue (le plus courant), la douleur, la nausée, la constipation et enfin, l'anxiété (le moins courant) (Figure 1). Les infirmières avaient le moins de confiance dans leur capacité à gérer les symptômes suivants, la confiance étant présentée dans l'ordre croissant : dépression, dysurie/ hématurie, anorexie, essoufflement et neuropathie; en revanche, elles avaient la plus grande confiance dans leur capacité à gérer la fièvre, la constipation, la nausée, les vomissements et la diarrhée. Les symptômes prioritaires en vertu de leur fréquence élevée et de la faible confiance du personnel infirmier incluaient l'anxiété, la neuropathie, la dépression, l'anorexie et les altérations cutanées. Les répondantes faisaient état des enjeux additionnels rapportés par les patients dans le cadre des services de soutien infirmier prodigués à distance. Les enjeux physiques comprenaient la tuméfaction, les éruptions cutanées, les saignements, les étourdissements, les troubles du sommeil, la toux et la xérostomie. Les défis de nature émotionnelle incluaient comment composer avec la colère, avec la détresse ou comment faire face à une maladie terminale. Les enjeux cognitifs comprenaient la confusion et les troubles de l'humeur induits par les stéroïdes. Des enjeux relatifs à l'intimité, à la sexualité et à l'image corporelle ont également été rapportés.

Attitudes, comportements, connaissances et niveau de confiance du personnel infirmier. Sur au moins 171 répondantes, la plupart étaient d'accord pour affirmer que les lignes directrices : étaient nécessaires (89\%), promouvaient la continuité $(93 \%)$ et amélioraient les soins aux patients (89\%). Quatrevingt-six infirmières (49\%) convenaient que leurs superviseurs attendaient d'elles qu'elles utilisent les lignes directrices. La plupart des infirmières étaient d'accord pour dire que les lignes directrices étaient en harmonie avec la pratique des médecins (46\%) et qu'elles avaient l'appui de l'équipe multidisciplinaire (60\%). La plupart des infirmières disposaient d'un accès aisé aux lignes directrices (70\%), 
utilisaient des lignes directrices pour gérer les symptômes (69\%), pouvaient facilement documenter leur utilisation des lignes directrices (69\%) et fournissaient de l'information écrite en vue de rehausser l'éducation aux patients (76\%). La plupart des infirmières ne pensaient pas que leur jugement clinique était limité par les lignes directrices (68\%), mais 121 infirmières (69\%) s'estimaient capables de gérer les symptômes sans lignes directrices. Seulement 93 infirmières (54\%) convenaient qu'elles avaient reçu suffisamment de formation en gestion des symptômes et 75 infirmières (43\%) jugeaient que les lignes directrices actuelles pour la gestion des symptômes ne convenaient pas pour les patients ayant des symptômes multiples et/ou complexes. Les infirmières étaient 117 (68\%) à affirmer qu'un accès électronique aux lignes directrices en augmenterait l'utilisation. Soixante-deux pour cent des infirmières ne se sentaient pas soumises à une pression constante pour faire en sorte que leurs interactions avec les patients restent brèves. Quarante-quatre infirmières (25\%) convenaient qu'elles ne disposaient pas d'assez de temps pour trouver et utiliser des lignes directrices.

Facilitateurs. L'analyse de contenu des réponses aux questions ouvertes a révélé les six principaux facilitateurs de la fourniture du soutien à distance (tableau 4). Beaucoup d'infirmières ont indiqué que pour faciliter l'utilisation de lignes directrices sur la gestion des symptômes, celles-ci devaient être accessibles, en format électronique, basés sur des données probantes et à jour. Le soutien à distance était facilité par : a) l'accès à des dossiers de santé électroniques renfermant une information actualisée; b) la réceptivité des médecins et des membres de l'équipe multidisciplinaire; c) un soutien administratif; et enfin, d) des ordinateurs disponibles et conviviaux dont le système de documentation est d'un emploi aisé, normalisé, électronique et a été formaté aux fins de la fourniture de soutien à distance. Deux répondantes ont signalé le besoin d'avoir des directives médicales à l'appui de la prescription des médicaments exigés pour la gestion des symptômes.

Obstacles éventuels. L'analyse de contenu a permis de dégager les huit principaux obstacles à la fourniture du soutien à distance (tableau 4). Cinquante infirmières (29\%) identifiaient les contraintes temporelles comme l'obstacle le plus important. Trente-six infirmières (21\%) cernaient le défi de l'obtention au téléphone de renseignements exacts sur les patients comme étant un obstacle important (p. ex. l'impossibilité de voir les patients, les problèmes de communication, l'exactitude de l'information relative aux antécédents médicaux et aux médicaments des patients). Pour vingt et une répondantes (12\%), la lourde charge de travail et la dotation insuffisante en personnel constituaient des défis. Vingt infirmières (11\%) cernaient des problèmes liés aux connaissances, à l'expérience, aux compétences d'évaluation et à la formation du personnel infirmier. Quatorze répondantes (8\%) faisaient état de l'insuffisance de l'accès aux services de soutien, notamment à ceux de diagnostic, de pharmacie, aux soins à domicile, aux médecins de famille et aux urgences. Cinq répondantes (3\%) décrivaient la difficulté que leur posait l'utilisation des lignes directrices existantes parce que les symptômes multiples et/ou complexes ne correspondaient pas aux lignes directrices actuelles portant chacune sur un seul symptôme. Deux répondantes (1\%) ont dégagé des défis liés à l'environnement notamment des milieux bruyants et des pratiques contradictoires de la part des médecins.

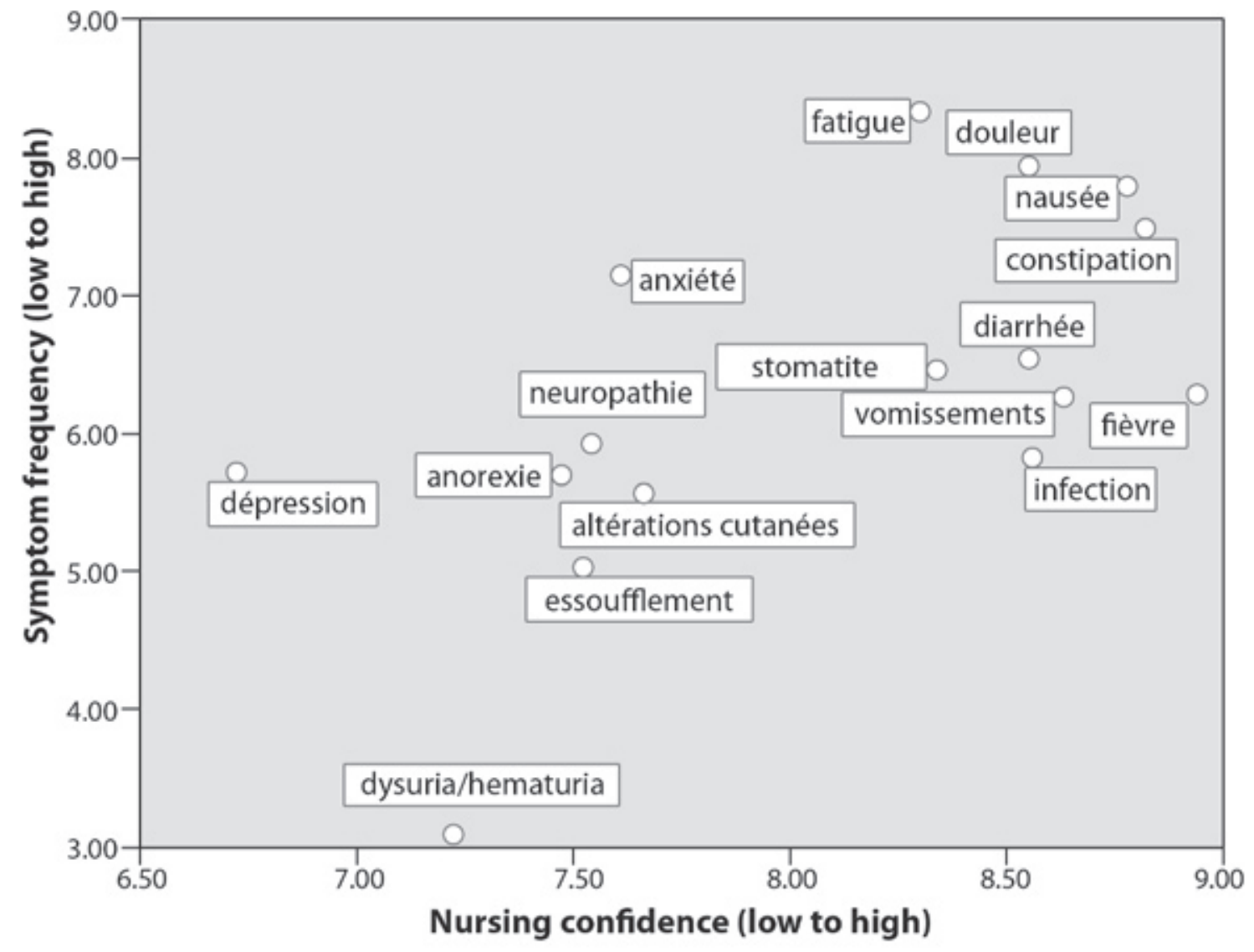

Figure 1 : Confiance des infirmières dans leurs aptitudes et fréquence des symptômes 


\section{Discussion}

À notre connaissance, il s'agit de la première étude visant à évaluer les perceptions des infirmières en oncologie relativement à la prévalence de symptômes, à leur degré de confiance envers leur capacité à gérer les symptômes à distance et enfin, les facteurs influant sur la fourniture du soutien à distance. La plupart des répondantes prodiguant un soutien à distance étaient des infirmières chevronnées dont l'expertise en oncologie avait été sanctionnée par la certification. Le soutien à distance était dispensé principalement au téléphone durant les heures d'ouverture régulières, du lundi au vendredi, et documenté par écrit dans le dossier de santé au moyen de formulaires papier. Les lignes directrices d'Action Cancer Ontario étaient celles qui étaient les plus utilisées, mais surtout à titre d'ouvrages de référence.

Quoique les infirmières aient signalé avoir de solides connaissances, avoir confiance dans leur capacité à gérer les symptômes et être animées d'attitudes positives envers l'utilisation de lignes directrices pour le traitement des symptômes, elles déterminaient qu'il leur fallait disposer de lignes directrices accessibles, en format électronique, à jour et basées sur des données probantes pour que le soutien à distance des symptômes soit facilité. En outre, les infirmières indiquaient que leur accès aux dossiers de santé électroniques des patients au moyen d'un système de documentation électronique accroîtrait l'utilisation des lignes directrices relatives aux symptômes. Ces résultats sont conformes à ceux de notre étude précédente qui révélait que les infirmières en oncologie sont plus susceptibles de documenter les appels lorsqu'elles ont accès aux dossiers de santé électroniques (Stacey et al., 2007). Des ressources accessibles et faciles d'emploi ainsi que des outils de documentation bien conçus favorisaient la dispensation de soins de qualité aux patients (Black, 2007). Les programmes liés aux centres d'appels de soins infirmiers ont des protocoles visant à guider la prise de décision des infirmières et disposent de systèmes de documentation électronique à l'appui (Stacey et al., 2003). Quoique ces ressources soient essentielles à la fourniture de télésoins de qualité (Ordre des infirmières et infirmières de l'Ontario, 2005), elles ne sont pas fournies de manière uniforme dans les programmes d'oncologie canadiens.

Des inquiétudes ont été soulevées quant à la menace que fait peser le recours aux lignes directrices et aux protocoles sur l'aspect «caring» des soins infirmiers (Wilson \& Hubert, 2002) et quant à la

Tableau 3: Attitudes, comportements, connaissances, niveau de confiance et perceptions des infirmières concernant les facteurs environnementaux entourant le soutien téléphonique à distance

\begin{tabular}{|c|c|c|c|c|}
\hline & $\mathbf{n}$ & $\begin{array}{c}\text { En désaccord } \\
\text { (\%) }\end{array}$ & $\begin{array}{c}\text { Neutre } \\
\text { (\%) }\end{array}$ & $\begin{array}{c}\text { D'accord } \\
\text { (\%) }\end{array}$ \\
\hline \multicolumn{5}{|l|}{ Attitude des infirmières envers les lignes directrices } \\
\hline Les lignes directrices favorisent la continuité & 175 & 2,8 & 4,6 & 92,6 \\
\hline Les lignes directrices améliorent les soins aux patients & 175 & 2,3 & 8,6 & 89,1 \\
\hline Les lignes directrices sont nécessaires & 173 & 2,9 & 8,1 & 89,0 \\
\hline Les lignes directrices sont soutenues par l'équipe multidisciplinaire & 175 & 11,4 & 29,1 & 59,5 \\
\hline Mes superviseurs exigent que j'utilise les lignes directrices & 174 & 20,7 & 29,9 & 49,4 \\
\hline Les lignes directrices sont en harmonie avec la pratique des médecins & 174 & 23,0 & 31,0 & 46,0 \\
\hline \multicolumn{5}{|l|}{ Comportements des infirmières } \\
\hline Je fournis de l'information afin de rehausser l'éducation des patients & 171 & 10,5 & 13,5 & 76,0 \\
\hline J'ai aisément accès à des lignes directrices basées sur des données probantes & 175 & 11,4 & 18,3 & 70,3 \\
\hline Il m'est facile de documenter & 173 & 16,1 & 15,0 & 68,9 \\
\hline J'utilise les lignes directrices pour gérer les symptômes & 173 & 11,0 & 20,2 & 68,8 \\
\hline \multicolumn{5}{|l|}{ Connaissances et niveau de confiance des infirmières } \\
\hline Je suis sûre de mes habiletés sans devoir compter sur des lignes directrices & 175 & 14,9 & 16,0 & 69,1 \\
\hline J'ai reçu suffisamment de formation en gestion des symptômes & 173 & 26,0 & 20,2 & 53,8 \\
\hline Les protocoles sont inappropriés pour les symptômes multiples/complexes & 175 & 24,0 & 33,1 & 42,9 \\
\hline Je n’ai pas de connaissances suffisantes pour gérer les symptômes & 174 & 92,0 & 4,6 & 3,4 \\
\hline Mon jugement clinique est limité par les lignes directrices & 174 & 67,8 & 28,8 & 3,4 \\
\hline J'ai appris ce que je sais auprès d'autres infirmières & 174 & 47,7 & 25,9 & 26,4 \\
\hline \multicolumn{5}{|l|}{ Les perceptions des infirmières relativement aux facteurs environnementaux } \\
\hline L'accès électronique en augmenterait l'utilisation & 173 & 8,1 & 24,3 & 67,6 \\
\hline J'éprouve des pressions constantes pour faire en sorte que les interactions soient brèves & 175 & 62,3 & 21,1 & 16,6 \\
\hline Je n'ai pas le temps de chercher et d'utiliser des lignes directrices & 175 & 44,0 & 30,9 & 25,1 \\
\hline
\end{tabular}


non-reconnaissance des préférences des patients individuels (Black, 2007; Stacey et al., 2007). Toutefois, les infirmières associées à cette étude manifestaient des attitudes positives vis-à-vis de l'emploi des lignes directrices. Ceci pourrait refléter l'évolution des attitudes envers les lignes directrices du fait de l'accès accru à ces types de ressources basées sur des données probantes et le fait qu'elles soient perçues comme des outils. Ou alors, ceci pourrait refléter un biais dans les réponses où les infirmières donnent les réponses qu'elles considèrent les plus appropriées plutôt que de faire état de la pratique actuelle.

Les obstacles les plus fréquemment mentionnés étaient la lourde charge de travail, les contraintes temporelles et d'importantes préoccupations relativement au problème de l'acquisition, au téléphone, d'informations exactes sur les patients. La lourde charge de travail et les contraintes temporelles font régulièrement partie des obstacles dégagés dans le cadre de toute initiative visant à modifier la pratique, et il est difficile de les régler de par elles-mêmes (Conley et al., 2010). L'exactitude des renseignements sur les patients obtenus dans le cadre du traitement symptomatique par téléphone s'accompagne de problèmes intrinsèques émanant de la faible information sensorielle (Black, 2007). Les infirmières ayant participé à cette étude dégageaient des facteurs qui pourraient atténuer ces obstacles. Par exemple, elles indiquaient à quel point il leur était difficile de comprendre les descriptions faites par les patients ou par leurs proches du problème en question. Des barrières linguistiques, des problèmes d'élocution ou des difficultés à entendre ce que disent les clients tandis quand les infirmières œuvrent dans leurs environnements de travail bruyants faisaient empirer ces situations. Selon les suggestions des infirmières de cette étude, ces problèmes pourraient être minimisés en ayant recours à des outils d'évaluation normalisés, en ayant accès aux dossiers des patient actualisés et disponibles par voie électronique et en fournissant des espaces de travail tranquilles. En outre, la formation en pratique infirmière dispensée au téléphone peut éventuellement mettre au point de nouvelles manières d'évaluer la gravité des symptômes lorsque l'ouïe est la seule information sensorielle sur laquelle on peut compter (Black, 2007; Stacey et al., 2007).

La nécessité d'une formation supplémentaire se reflétait dans la rétroaction qualitative des infirmières en oncologie chevronnées interrogées. Il est intéressant de noter le manque de consensus dans la littérature sur les compétences que devraient posséder les infirmières se livrant au triage téléphonique. Par exemple, Black (2007) suggère que les infirmières ont besoin d'avoir au minimum de 3 à 5 années d'expérience variée des soins infirmiers en oncologie ainsi qu'une habileté en relations humaines et une habileté en communication dépassant la moyenne. Conley et ses collègues (2010) recommandent un niveau établi de compétence pour toutes les infirmières en oncologie assumant un rôle de triage téléphonique selon une démarche normalisée et fondée sur des éléments probants relativement à l'évaluation des patients, à leur suivi et à la documentation des appels. Dans une étude qualitative explorant les défis déontologiques auxquels faisaient face les infirmières fournissant des soins infirmiers au téléphone, Holmstrom et Hoglund (2007) ont constaté que l'autonomie, l'intégrité et la prioritisation étaient les enjeux importants. De plus, le développement de la compétence éthique peut potentiellement aider à réduire l'incertitude et la détresse morales par les télé-infirmières. Les infirmières de la présente étude ont souligné la nécessité de la formation sur le plan de l'utilisation des lignes directrices sur les symptômes et de la gestion de symptômes multiples (Holmstrom \& Hoglund, 2007).

Les lignes directrices actuelles sont inappropriées pour les infirmières dispensant des soins à des patients en oncologie éprouvant des symptômes complexes et/ou multiples. Aux dires des infirmières en oncologie, les lignes directrices qu'elles utilisaient le plus souvent étaient celles disponibles en version papier, ce qui signifie qu'elles se limitent à la présentation de symptômes individuels. Leur emploi en cas de symptômes multiples exige des infirmières qu'elles identifient le symptôme le plus sévère ou incommodant

Tableau 4 : Obstacles et facilitateurs obtenus qualitativement $(n=175)$

\section{Obstacles}

Temps (50)

- Contraintes temporelles

Difficulté d'obtenir des renseignements exacts (36)

- Difficulté d'obtenir des renseignements exacts sur le symptôme (11)

- L'impossibilité de voir les patients (10)

- Défis de communication: langue, ouïe, énonciation (8)

- Information nécessaire sur les antécédents médicaux, les

médicaments consommés (5)

- Le fait de ne pas connaître les patients (2)

Charge de travail (21)

- Lourde charge de travail, dotation en personnel insuffisante

Attributs liés aux soins infirmiers (20)

- Manque de connaissances, d'expérience, de compétences en

évaluation (12)

- Manque de formation (8)

Services de soutien (14)

- Besoin d'avoir accès aux services de diagnostic, de pharmacie, soins à domicile, médecins de famille et urgences

Complexité des symptômes (5)

- Les symptômes multiples/complexes ne correspondent pas aux lignes directrices

Environnement (2)

- Bruit ambiant

Attributs liés à l'équipe (2)

- Pratique non uniforme

- Préférences particulières des médecins
Facilitateurs

Accès à des lignes directrices sur les symptômes (22)

- Aisément accessibles, électroniques, actualisées, basées sur des données probantes (18)

- Nécessité d'avoir à l'appui du matériel d'éducation aux patients (3)

- Disponibles en français (1)

Dossiers de santé électroniques (16)

- Aisément accessibles

- Doivent être actuels en termes de documentation et de résultats d'examens

Attributs liés à l'équipe (11)

- Disponibilité, réceptivité et appui des médecins

- Soutien de l'équipe multidisciplinaire

- Soutien des administrateurs

Technologie-ordinateurs (9)

- Aisément accessibles, disponibles, conviviaux

Documentation (7)

- Facile d'emploi

- Suffisamment de temps pour la rédiger

- Normalisée

- Formatée pour le soutien téléphonique

- Électronique

Modèle de soins (3)

- Modèle de soins intégraux

Directives médicales (2)

- Exigées pour faciliter la prescription des médicaments liés à la gestion des symptômes

Nombre de commentaires entre parenthèses 
afin de sélectionner le protocole individuel qui lui correspond. Cependant, il est possible que les personnes cancéreuses présentant des symptômes multiples n'éprouvent pas des symptômes interdépendants et qu'ils aient donc besoin d'une approche plus globale en matière de gestion des symptômes (Barsevick, 2007; Miakowski, Dodd \& Lee, 2004). Un problème de taille est que les lignes directrices existantes n'examinent pas le fait que des symptômes multiples peuvent partager des étiologies communes ni le fait que maîtriser un symptôme tel que la douleur peut avoir une incidence positive sur un autre symptôme tel que le sommeil. Lors de leur formation, les infirmières n'étudient pas spécifiquement comment évaluer la gravité des symptômes quand plusieurs symptômes multiples coexistent. Les limites de la mise en ouvre de protocoles normalisés incluaient le fait que les patients ne se conforment pas toujours aux algorithmes prédéterminés, ce qui minimise les aspects de la situation directement liés aux patients (Black, 2007).

Plusieurs limites ont été dégagées au niveau de la présente étude. La généralisabilité de ces résultats doit être considérée dans leur contexte. La plupart des répondantes à l'étude étaient des infirmières en oncologie chevronnées ouvrant dans le système de soins de santé public. Il se peut donc que leurs réponses ne reflètent pas les opinions d'infirmières en oncologie moins expérimentées ou de celles travaillant dans des milieux de soins non canadiens. Ensuite, étant données certaines contradictions entre les réponses quantitatives et les réponses qualitatives, il est possible que certaines infirmières aient donné les réponses qui, d'après elles, étaient les «bonnes» réponses plutôt que d'exprimer leurs propres perspectives.

\section{Conclusion}

Les infirmières en oncologie sondées dans le cadre de cette étude fournissaient principalement un soutien téléphonique à distance pour la gestion des symptômes durant les heures d'ouverture régulières en

\section{RÉFÉRENCES}

Barbera, L., Seow, H., Howell, D., Sutradhar, R., Earle, C., Liu, Y., et al. (2010). Symptom burden and performance status in a populationbased cohort of ambulatory cancer patients. Cancer, 116(24), 5767-5776.

Barsevick, A.M. (2007). The elusive concept of the symptom cluster. Oncology Nursing Forum, 34(5), 971-980.

Black, K.L. (2007). Standardization of telephone triage in pediatric oncology. Journal of Pediatric Oncology Nursing, 24(4), 190-199.

Conley, S.B., O’Hanlon Curry, J., Hines, M., Baker, K., Schmidt, K., Zwier, K., Siever B., ... Kline, N.E. Association of Pediatric Hematology/ Oncology Nursing. (2010). Consensus statements: Ambulatory pediatric oncology nursing practice. Journal of Pediatric Oncology Nursing, 27(1), 6-9.

Dillman, D.A. (2000). Mail and Internet surveys: The tailored design. New York: John Wiley \& Sons.

Dodd, M., Janson, S., Facione, N., Faucett, J., Froelicher, E. S., Humphreys, J., et al. (2001). Advancing the science of symptom management. Journal of Advanced Nursing, 33(5), 668-676.

Dodd, M.J., Miaskowski, C., \& Lee, K.A. (2004). Occurrence of symptom clusters. Journal of the National Cancer Institute, Monographs(32), 76-78.

Goodwin, S. (2007). Telephone nursing: An emerging practice area. Nursing Leadership, 20(4), 37-45.

Graham, I., Harrison, M.B., Moffat, C., \& Franks, P. (2001). Leg ulcer care: Nursing attitudes and knowledge. The Canadian Nurse, 97(3), 19-24.

Graham. I., \& Logan, J. (2004). Innovations in knowledge transfer and continuity of care. Canadian Journal of Nursing Research, 36, 89103.

Holmstrom, I., \& Hoglund, A.T. (2007). The faceless encounter: Ethical dilemmas in telephone nursing. Journal of Clinical Nursing, 16(10), 1865-1871. utilisant les lignes directrices sur les symptômes individuels à titre d'ouvrages de référence. Les infirmières manifestaient des attitudes positives à l'égard de l'accès à des lignes directrices actualisées et basées sur des données probantes tout en ajoutant que pour que celles-ci soient utiles, il fallait qu'elles disposent d'applications électroniques reliées à la documentation figurant dans les dossiers de santé électroniques des patients. Pour les concepteurs de lignes directrices, les priorités cliniques incluent la gestion des symptômes pour l'anxiété, les neuropathies, l'anorexie, la dépression et les altérations cutanées. En outre, il est nécessaire d'établir des lignes directrices et de la formation afin d'aider les infirmières sur le plan de l'identification, de la prioritisation et de la gestion des symptômes concomitants. Les symptômes qui surviennent ainsi ensemble peuvent partager des étiologies sous-jacentes communes et donc être soulagés par des stratégies de gestion similaires. En plus des contraintes temporelles et du volume de travail, les infirmières ont souligné les défis liés à l'obtention, au téléphone, de renseignements exacts sur les patients. Des recherches additionnelles devront être menées pour normaliser les critères guidant l'évaluation à distance des patients et pour évaluer la rigueur empirique des lignes directrices utilisées dans le cadre de la gestion à distance des symptômes. Finalement, il est nécessaire d'établir des liens entre l'utilisation de lignes directrices normalisées basées sur des données probantes d'une part, et des résultats positifs pour les patient, d'autre part, puisque fort peu de recherches ont été faites dans ce domaine jusqu'à présent (Mayo et al., 2002; Wilson \& Hubert, 2002).

\section{Remerciements et financement}

Cette enquête est une des études menées dans le cadre de COSTaRS (Système pancanadien de triage des symptômes oncologiques et des soins de soutien à distance). Le Partenariat canadien contre le cancer (le Partenariat) a fourni un financement à l'appui de ce projet.

Mayo, A.M., Chang, B.L., \& Omery, A. (2002). Use of protocols and guidelines by telephone nurses. Clinical Nursing Research, 11(2), 204-219.

Miaskowski, C., Dodd, M., \& Lee, K. (2004). Symptom clusters: The new frontier in symptom management research. Journal of the National Cancer Institute, Monographs, (32), 17-21.

Molassiotis, A., Wengstrom, Y., \& Kearney, N. (2010). Symptom cluster patterns during the first year after diagnosis with cancer. Journal of Pain \& Symptom Management, 39(5), 847-858.

Ordre des infirmières et infirmiers de l'Ontario, Directives professionnelles (2005). Les télésoins. Ottawa: Ordre des infirmières et infirmiers de l'Ontario. Pub. No 51041.

Richard, M.L., Parmar, M.P., Calestagne, P.P., \& McVey, L. (2010). Seeking patient feedback: An important dimension of quality in cancer care. Journal of Nursing Care Quality, 25(4), 344-351.

Stacey, D., Bakker, D., Green, E., Zanchetta, M., \& Conlon, M. (2007). Les services de soins infirmiers téléphoniques en oncologie ambulatoire: une enquête provinciale. Revue canadienne de soins infirmiers en oncologie, 17(4): 186(é1.-5). [Article offert en ligne]

Stacey, D., Graham, I., O’Connor, A.M., \& Pomey, M.P. (2005). Barriers and facilitators influencing call centre nurses' decisions support for callers facing values-sensitive decisions: A mixed methods study. Worldviews on Evidence-Based Nursing, 2(4), 184-195.

Stacey, D., Noorani, H., Fisher, A., Robinson, D., Joyce, J., \& Pong, R. (2003). Le télétriage: étude méthodique et enquête auprès des centres d'appels canadiens. Ottawa: Office canadien de coordination de l'évaluation des technologies de la santé.

Wilson, R., \& Hubert, J. (2002). Resurfacing the care in nursing by telephone: Lessons from ambulatory oncology. Nursing Outlook, 50(4), 160-164. 